\title{
Review of: "Detection and quantification of Covid-19 antiviral drugs in biological fluids and tissues"
}

Sophia Trantza

Potential competing interests: The author(s) declared that no potential competing interests exist.

This is a very well written article that adds help to the scientific society for COVID-19 and needs to be published. The authors should be careful of references in some parts, some minor language issues and italics when they refer to other publications, as this is a review article. Please see comments in the supplementary data provided. 